\title{
Uni-Vector-Sensor Dimensionality Reduction MUSIC Algorithm for DOA and Polarization Estimation
}

\author{
Lanmei Wang, ${ }^{1}$ Le Yang, ${ }^{1}$ Guibao Wang, ${ }^{2}$ Zhihai Chen, ${ }^{1}$ and Minggao Zou ${ }^{1}$ \\ ${ }^{1}$ School of Physics and Optoelectronic Engineering, Xidian University, Xi'an 710071, China \\ ${ }^{2}$ National Laboratory of Radar Signal Processing, Xidian University, Xian 710071, China \\ Correspondence should be addressed to Lanmei Wang; lmwang@mail.xidian.edu.cn
}

Received 23 May 2014; Revised 20 October 2014; Accepted 1 November 2014; Published 19 November 2014

Academic Editor: Tiedong Ma

Copyright (c) 2014 Lanmei Wang et al. This is an open access article distributed under the Creative Commons Attribution License, which permits unrestricted use, distribution, and reproduction in any medium, provided the original work is properly cited.

\begin{abstract}
This paper addresses the problem of multiple signal classification- (MUSIC-) based direction of arrival (DOA) and polarization estimation and proposes a new dimensionality reduction MUSIC (DR-MUSIC) algorithm. Uni-vector-sensor MUSIC algorithm provides estimation for DOA and polarization; accordingly, a four-dimensional peak search is required, which hence incurs vast amount of computation. In the proposed DR-MUSIC method, the signal steering vector is expressed in the product form of arrival angle function matrix and polarization function vector. The MUSIC joint spectrum is converted to the form of Rayleigh-Ritz ratio by using the feature where the 2-norm of polarization function vector is constant. A four-dimensional MUSIC search reduced the dimension to two two-dimensional searches and the amount of computation is greatly decreased. The theoretical analysis and simulation results have verified the effectiveness of the proposed algorithm.
\end{abstract}

\section{Introduction}

1.1. Review of Relevant Literature. The estimation of direction of arrival (DOA) using sensor data processing has attracted a significant attention lately. The existing methods for estimating DOA include maximum likelihood (ML) approach $[1,2]$ and trilinear decomposition algorithm [3-6] as well as computationally more efficient techniques such as MUSIC (multiple signal classification) [7-9], ESPRIT (estimation of signal parameters via rotational invariance techniques) [1021], and others [22-25]. Electromagnetic vector sensor arrays have some inherent advantages over scalar arrays because of their polarization characteristics. Hence the advantages of different electromagnetic vector sensor arrays are being explored. The problem of estimating signal polarizations along with arrival angles has been discussed previously in many articles [3-6, 10-29]. The first direction finding algorithms explicitly exploiting all six electromagnetic components seem to have been developed separately by Nehorai and Paldi [26] and Li [27]. Nehorai and Paldi [26], who coined the term vector sensor, pioneered the simple but novel idea that estimated directly the two-dimensional radial direction of a source by using the vector cross product of the electric-field and the magnetic-field vector estimates. Li [27] developed the ESPRIT algorithm for a vector-sensor array. The cross-product-based DOA estimation algorithm was first adapted to ESPRIT by Wong and Zoltowski [10-12], which utilizes extended inter-vector-sensor spatial invariances in a multielement planar array of vector sensors. Wong and Zoltowski [12] further developed a closed-form direction finding algorithm applicable to multiple arbitrarily spaced vector sensors at possibly unknown locations. MUSIC is the attractive subspace algorithm for estimating DOA and polarization, because MUSIC produces a moderate signal-to-noise (SNR) estimation performance comparable to the optimal methods and is also applicable to irregularly spaced arrays. MUSIC algorithm was first put forward by Schmidt [8] and MUSIC-based algorithms for DOA and polarization problem were proposed by Wong et al. [28, 29], who also developed uni-vector-sensor ESPRIT algorithm [13]. But to explore polarization in MUSIC using six-component electromagnetic vector sensor, which provides estimation for both DOA and polarization, a $4 \mathrm{D}$ peak search is required, which incurs vast amount of computation. Reduced-dimension MUSIC methods for the angle and polarization estimation have been investigated in [28-33], which requires prior conditions. 
The authors in [28] have studied the self-initiating MUSIC DOA and polarization estimation algorithm, which can decouple the DOA estimation problem from the polarization estimation problem, whose work uses ESPRIT on pairs of vector sensors to self-generate coarse estimates of the arrival angles to start off its MUSIC-based iterative search. In [29], a novel polynomial-rooting-based algorithm for multiple-source DOA and polarization estimation method is proposed, which needs to pair two sets of estimates. In [30, 31], the local extreme points are obtained by implementing the derivative of MUSIC spectrum function. The premise of this method is that the MUSIC spectrum function is the convex function. Nevertheless, in many cases it is difficult to satisfy this condition. In [32], Lagrange multiplier method is proposed, which requires that the optimization function is a convex function. In [33], the authors propose a beamspace MUSIC algorithm, assuming that $\mathrm{K}$ plane waves impinge at a fixed elevation angle and the mutual coupling matrix is the function of azimuth.

1.2. The Basic Idea. In this paper, we propose a novel dimensionality reduction MUSIC (DR-MUSIC) approach to DOA and polarization estimation that overcomes the multidimensional searching shortcomings and requires no a priori source information. Our proposed method represents the subspace (MUSIC-based) method that estimates the DOA and polarization of multiple sources using a single vector sensor. Such sensor structures correspond to numerous practical situations where a six-component vector sensor is used. The procedure of the proposed method is as follows. Firstly, we introduce a novel Rayleigh-Ritz theorem that is applicable to MUSIC dimensionality reduction whose sensor is a single vector sensor. Secondly, the signal steering vector is represented as the product of the arrival angle function matrix and polarization function vector; the MUSIC joint spectrum is converted to the Rayleigh-Ritz ratio according to the feature of polarization function vector. Finally, using the Rayleigh-Ritz theorem, the proposed method can reduce MUSIC's iterative search from four dimensions to two dimensions, which greatly decrease the amount of computation. Hence, this proposed algorithm (1) only needs two twodimensional searches and it has lower complexity; (2) can be suitable for irregular array geometry; (3) has the advantage of parameter automatic matching and avoids multidimensional searching. The simulation results show that the proposed method can effectively reduce the complexity of MUSIC searching. The paper is organized as follows. In Section 2, a mathematical theory of Hermitian matrix and its relevant properties are presented. Section 3 introduces the signal model for a single six-component vector sensor. In Section 4, the conventional MUSIC-based algorithm is introduced. In Section 5, a dimensionality reduction MUSIC (DR-MUSIC) method is derived for uni-vector-sensor, and the problem of multidimensional searching is solved. In Section 6, the computational complexity analysis of the proposed algorithm is given. Simulation results are presented in Section 7. In Section 8, conclusions are given.

\section{Hermitian Matrix and Relevant Properties}

In mathematics, a matrix $\mathbf{A}=\left[a_{i j}\right] \in \mathbf{M}_{n}$ is said to be Hermitian if $\mathbf{A}=\mathbf{A}^{\mathrm{H}}$ where $\mathbf{A}^{\mathrm{H}}$ is the conjugate transpose of the matrix $\mathbf{A}$. It is skew-Hermitian if $\mathbf{A}=-\mathbf{A}^{\mathrm{H}}$. Hermitian matrix is also called self-adjoint matrix. Let $\mathbf{A} \in \mathbf{M}_{n}$ be Hermitian. Then

(1) $\mathbf{x}^{\mathrm{H}} \mathbf{A x}$ is real for all $\mathbf{x} \in \mathrm{C}^{n}$;

(2) all the eigenvalues of $\mathbf{A} \in \mathbf{M}_{n}$ are real;

(3) $\mathbf{S}^{\mathrm{H}} \mathbf{A S}$ is Hermitian for all $\mathbf{S} \in \mathbf{M}_{n}$;

(4) the entries on the main diagonal (top left to bottom right) are real;

(5) the determinant of a Hermitian matrix is real.

Hermitian matrix has the following important properties.

Property 1 (the spectral theorem for Hermitian matrices). Let $\mathbf{A} \in \mathbf{M}_{n}$ be given. Then $\mathbf{A} \in \mathbf{M}_{n}$ is Hermitian if and only if there are a unitary matrix $\mathbf{U} \in \mathbf{M}_{n}$ and a real diagonal matrix $\boldsymbol{\Lambda} \in \mathbf{M}_{n}$ such that $\mathbf{A}=\mathbf{U} \boldsymbol{\Lambda} \mathbf{U}^{\mathrm{H}}$. Moreover, $\mathbf{A}$ is real and Hermitian if and only if there are a real orthogonal matrix $\mathbf{P} \in \mathbf{M}_{n}$ and a real diagonal matrix $\boldsymbol{\Lambda} \in \mathbf{M}_{n}$ such that $\mathbf{A}=\mathbf{P} \boldsymbol{\Lambda} \mathbf{P}^{\mathrm{H}}$.

Property 2 (the variational characterization of eigenvalues of Hermitian matrices). The eigenvalues of Hermitian matrices can be characterized as the solutions of optimization problems. Since the eigenvalues of a Hermitian matrix $\mathbf{A} \in \mathbf{M}_{n}$ are real, they are labeled according to increasing (nondecreasing) size:

$$
\lambda_{\text {min }}=\lambda_{1} \leq \lambda_{2} \leq \cdots \leq \lambda_{n-1} \leq \lambda_{n}=\lambda_{\max }
$$

The smallest and largest eigenvalues are easily characterized as the solutions to constrained minimum and maximum problem. The characterization theorem bears the names of two British physicists, and the expression $\mathbf{x}^{\mathrm{H}} \mathbf{A x} / \mathbf{x}^{\mathrm{H}} \mathbf{x}$ is known as a Rayleigh-Ritz ratio.

\section{Signal and Array Models}

Consider a six-component electromagnetic vector sensor, which consists of three spatially colocated identical but orthogonally oriented, electrically short dipoles and magnetically small loops separately measuring all three electricfield components and three magnetic-field components of incident signals, as shown in Figure 1.

Assume that the $k$ th $(1 \leq k \leq K)$ narrow-band, transverse electromagnetic (TEM) plane-wave signal having travelled through a nonconductive homogenous medium impinges upon the receiving sensor. For an incoming unit-power signal, an electromagnetic vector sensor manifold would be 


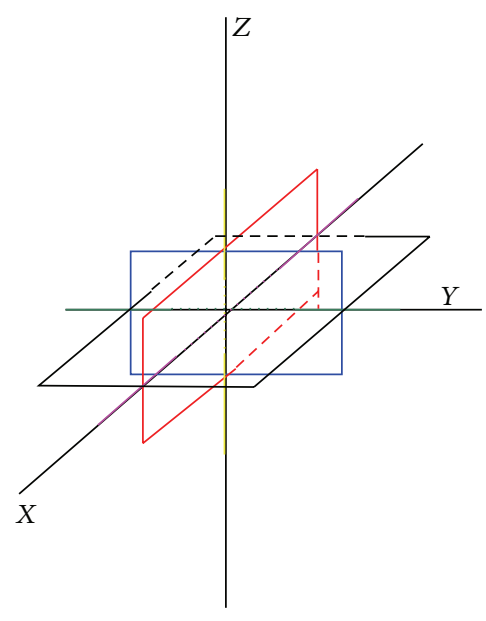

Figure 1: A six-component electromagnetic vector sensor.

a concatenation of the $3 \times 1$ electric-field vector $\mathbf{e}$ with the $3 \times 1$ magnetic-field vector $\mathbf{h}$; it can be expressed as [13]

$$
\begin{aligned}
\mathbf{a}_{k} & =\left[\begin{array}{c}
e_{k x} \\
e_{k y} \\
e_{k z} \\
h_{k x} \\
h_{k y} \\
h_{k z}
\end{array}\right] \\
= & \underbrace{\left[\begin{array}{cc}
\cos \theta_{k} \cos \phi_{k} & -\sin \phi_{k} \\
\cos \theta_{k} \sin \phi_{k} & \cos \phi_{k} \\
-\sin \theta_{k} & 0 \\
-\sin \phi_{k} & -\cos \theta_{k} \cos \phi_{k} \\
\cos \phi_{k} & -\cos \theta_{k} \sin \phi_{k} \\
0 & \sin \theta_{k}
\end{array}\right]}_{\Omega\left(\theta_{k}, \phi_{k}\right)} \underbrace{\mathrm{g}\left(\gamma_{k}, \eta_{k}\right)}_{\left.\begin{array}{c}
\sin \gamma_{k} \mathrm{e}^{\mathrm{j} \eta_{k}} \\
\cos \gamma_{k}
\end{array}\right]}
\end{aligned}
$$

where $\theta_{k} \in[0, \pi]$ is the signals elevation angle measured from the positive $z$-axis, $\phi_{k} \in[0,2 \pi]$ denotes the azimuth angle measured from the positive $x$-axis, $\gamma_{k} \in[0, \pi / 2]$ represents the auxiliary polarization angle, and $\eta_{k} \in[-\pi, \pi]$ symbolizes the polarization phase difference. $\Omega\left(\theta_{k}, \phi_{k}\right)$ and $\mathbf{g}\left(\gamma_{k}, \eta_{k}\right)$ are, respectively, the DOA function matrix and the polarization function vector.

\section{MUSIC Algorithm}

$K(K \leq 5)$ narrow-band transverse electromagnetic (TEM) plane-wave signals impinge upon such a six-component electromagnetic vector sensor; the received data can be represented as

$$
\mathbf{X}(t)=\mathbf{A S}(t)+\mathbf{N}(t)
$$

where $\mathbf{X}(t), \mathbf{S}(t), \mathbf{N}(t)$, and $\mathbf{A}=\left[\mathbf{a}_{1}, \ldots, \mathbf{a}_{K}\right]$ are, respectively, the received data, the $K$ uncorrelated incident signals, the zero-mean additive complex Gaussian noise at time $t$, and the steering vector matrix of incident signals. Here the number of signals $K$ is assumed to be known. If not, we can get the value of $K$ by MDL principle.
The correlation matrix of received data $\mathbf{X}(t)$ is given by

$$
\mathbf{R}_{x}=\mathrm{E}\left[\mathbf{X X}^{\mathrm{H}}\right]=\mathbf{A R}_{s} \mathbf{A}^{\mathrm{H}}+\sigma^{2} \mathbf{I}
$$

with $\mathbf{R}_{x}$ representing the $6 \times 6$ matrix, $\mathrm{E}[\cdot]$ symbolizing the statistical mean, $(\cdot)^{\mathrm{H}}$ denoting the complex conjugate transpose, $\sigma^{2}$ indicating the white noise power, and $\mathbf{R}_{s}=$ $\mathbf{E}\left[\mathbf{s}\left(t_{1}\right) \mathbf{s}^{\mathrm{H}}\left(t_{1}\right)\right]$ being the $K \times K$ source covariance matrix.

Let $\mathbf{U}_{s}$ be the $6 \times K$ matrix composed of the $K$ eigenvectors corresponding to the $K$ largest eigenvalues of $\mathbf{R}_{x}$ and let $\mathbf{U}_{n}$ denote the $6 \times(6-K)$ matrix composed of the remaining $6-K$ eigenvectors of $\mathbf{R}_{x}$. The MUSIC joint spectrum function of $4 \mathrm{D}$ MUSIC algorithm can be expressed in terms of the noise eigenvectors:

$$
\mathbf{P}=\frac{1}{\mathbf{a}^{\mathrm{H}} \mathbf{U}_{n} \mathbf{U}_{n}^{\mathrm{H}} \mathbf{a}},
$$

where a denotes $\mathbf{a}(\theta, \phi, \gamma, \eta)$. The MUSIC employs the function (5) and picks the locations of $K$ largest peaks of $\mathbf{P}$. Thus, the objective function for maximization is

$$
\mathbf{P}_{\text {music }}\left(\widehat{\theta}_{k}, \widehat{\phi}_{k}, \widehat{\gamma}_{k}, \widehat{\eta}_{k}\right)=\max _{\theta, \phi, \gamma, \eta}\left(\frac{1}{\mathbf{a}^{\mathrm{H}} \mathbf{U}_{n} \mathbf{U}_{n}^{\mathrm{H}} \mathbf{a}}\right) .
$$

As an alternative (6) can be expressed as follows:

$$
\mathbf{P}_{\text {music }}\left(\widehat{\theta}_{k}, \widehat{\phi}_{k}, \widehat{\gamma}_{k}, \widehat{\eta}_{k}\right)=\frac{1}{\min _{\theta, \phi, \gamma, \eta}\left(\mathbf{a}^{\mathrm{H}} \mathbf{U}_{n} \mathbf{U}_{n}^{\mathrm{H}} \mathbf{a}\right)} .
$$

Theoretically, the DOA and polarization estimation in formula (7) can be estimated by bringing a $4 \mathrm{D}$ search to bear on the ranges of all parameters, however, which is extremely computation exhaustive.

\section{DR-MUSIC Algorithm}

To reduce the amount of calculation, the dimensionality reduction algorithm must be thoroughly studied. The signal steering vector is expressed as the product of arrival angle function matrix and polarization function vector; that is,

$$
\mathbf{a}(\theta, \phi, \gamma, \eta)=\mathbf{\Omega}(\theta, \phi) \mathbf{g}(\gamma, \eta) .
$$

For the sake of simplicity, we also let $\Omega$ denote $\Omega(\theta, \phi)$ and let $\mathbf{g}$ denote $\mathbf{g}(\gamma, \eta)$. From (2), polarization function vector $\mathbf{g}$ meets the relationship of $\mathbf{g}^{\mathrm{H}} \mathbf{g}=1$ and thus the MUSIC-based spectrum function can be expressed as

$$
\begin{aligned}
& \mathbf{P}_{\text {music }}\left(\widehat{\theta}_{k}, \widehat{\phi}_{k}, \widehat{\gamma}_{k}, \widehat{\eta}_{k}\right) \\
& \quad=\left[\min _{\theta, \phi, \gamma, \eta} \frac{\mathbf{a}^{\mathrm{H}} \mathbf{U}_{n} \mathbf{U}_{n}^{\mathrm{H}} \mathbf{a}}{1}\right]^{-1}=\left[\min _{\theta, \phi, \gamma, \eta} \frac{\mathbf{g}^{\mathrm{H}} \mathbf{\Omega}^{\mathrm{H}} \mathbf{U}_{n} \mathbf{U}_{n}^{\mathrm{H}} \mathbf{\Omega} \mathbf{g}}{\mathbf{g}^{\mathrm{H}} \mathbf{g}}\right]^{-1} .
\end{aligned}
$$

Let $\mathbf{B}(\theta, \phi)=\mathbf{\Omega}^{\mathrm{H}} \mathbf{U}_{n} \mathbf{U}_{n}^{\mathrm{H}} \boldsymbol{\Omega}$; as an alternative (9) can be shown as follows:

$$
\mathbf{P}_{\text {music }}\left(\widehat{\theta}_{k}, \widehat{\phi}_{k}, \widehat{\gamma}_{k}, \widehat{\eta}_{k}\right)=\left[\min _{\theta, \phi, \gamma, \eta} \frac{\mathbf{g}^{\mathrm{H}} \mathbf{B}(\theta, \phi) \mathbf{g}}{\mathbf{g}^{\mathrm{H}} \mathbf{g}}\right]^{-1},
$$


where $\mathbf{B}(\theta, \phi)$ is the self-adjoint matrix, namely, Hermitian matrix. The optimization problem in formula (10) is translated into the solutions to a minimum problem of RayleighRitz ratio. In the next section, we introduce Rayleigh-Ritz theorem [34] for the solution of the minimization problem in (10).

Rayleigh-Ritz Theorem. Let $\mathbf{B} \in \mathbf{M}_{n}$ be Hermitian matrix (in this paper $n=2)$, and let the eigenvalues of $\mathbf{B}$ be ordered as in (1); then

$$
\begin{gathered}
\lambda_{1} \mathbf{g}^{\mathrm{H}} \mathbf{g} \leq \mathbf{g}^{\mathrm{H}} \mathbf{B} \mathbf{g} \leq \lambda_{n} \mathbf{g}^{\mathrm{H}} \mathbf{g} \quad \mathbf{g} \in \mathrm{C}^{n}, \\
\lambda_{\max }=\lambda_{n}=\max _{\mathbf{g} \neq \mathbf{0}}\left(\frac{\mathbf{g}^{\mathrm{H}} \mathbf{B} \mathbf{g}}{\mathbf{g}^{\mathrm{H}} \mathbf{g}}\right)=\max _{\mathbf{g}^{\mathrm{H}} \mathbf{g}=1}\left(\mathbf{g}^{\mathrm{H}} \mathbf{B g}\right), \\
\lambda_{\min }=\lambda_{1}=\min _{\mathbf{g} \neq \mathbf{0}} \frac{\mathbf{g}^{\mathrm{H}} \mathbf{B} \mathbf{g}}{\mathbf{g}^{\mathrm{H}} \mathbf{g}}=\min _{\mathbf{g}^{\mathrm{H}} \mathbf{g}=1} \mathbf{g}^{\mathrm{H}} \mathbf{B g} .
\end{gathered}
$$

Proof. Since $\mathbf{B}$ is Hermitian matrix, there is a unitary matrix $\mathbf{U} \in \mathbf{M}_{n}$ such that $\mathbf{B}=\mathbf{U} \boldsymbol{\Lambda} \mathbf{U}^{\mathrm{H}}$ with $\boldsymbol{\Lambda}=\operatorname{diag}\left(\lambda_{1}, \lambda_{2}, \ldots, \lambda_{n}\right)$. For any $\mathbf{g} \in \mathrm{C}^{n}$ we have

$$
\mathbf{g}^{\mathrm{H}} \mathbf{B} \mathbf{g}=\mathbf{g}^{\mathrm{H}} \mathbf{U} \boldsymbol{\Lambda} \mathbf{U}^{\mathrm{H}} \mathbf{g}=\left(\mathbf{U}^{\mathrm{H}} \mathbf{g}\right)^{\mathrm{H}} \boldsymbol{\Lambda}\left(\mathbf{U}^{\mathrm{H}} \mathbf{g}\right)=\sum_{i=1}^{n} \lambda_{i}\left|\left(\mathbf{U}^{\mathrm{H}} \mathbf{g}\right)_{i}\right|^{2} .
$$

Since each term $\left|\left(\mathbf{U}^{\mathrm{H}} \mathbf{g}\right)_{i}\right|^{2}$ is nonnegative, we have

$$
\begin{aligned}
\lambda_{\min } \sum_{i=1}^{n}\left|\left(\mathbf{U}^{\mathrm{H}} \mathbf{g}\right)_{i}\right|^{2} & \leq \mathbf{g}^{\mathrm{H}} \mathbf{B g}=\sum_{i=1}^{n} \lambda_{i}\left|\left(\mathbf{U}^{\mathrm{H}} \mathbf{g}\right)_{i}\right|^{2} \\
& \leq \lambda_{\max } \sum_{i=1}^{n}\left|\left(\mathbf{U}^{\mathrm{H}} \mathbf{g}\right)_{i}\right|^{2} .
\end{aligned}
$$

Because $\mathbf{U}$ is unitary,

$$
\sum_{i=1}^{n}\left|\left(\mathbf{U}^{\mathrm{H}} \mathbf{g}\right)_{i}\right|^{2}=\sum_{i=1}^{n}\left|\mathbf{g}_{i}\right|^{2}=\mathbf{g}^{\mathrm{H}} \mathbf{g}
$$

And hence we have shown that

$$
\lambda_{1} \mathbf{g}^{\mathrm{H}} \mathbf{g}=\lambda_{\min } \mathbf{g}^{\mathrm{H}} \mathbf{g} \leq \mathbf{g}^{\mathrm{H}} \mathbf{B g} \leq \lambda_{\max } \mathbf{g}^{\mathrm{H}} \mathbf{g}=\lambda_{n} \mathbf{g}^{\mathrm{H}} \mathbf{g} .
$$

According to formula (13), (10) can be expressed in DRMUSIC spatial spectrum form as listed below:

$$
\mathbf{P}_{\text {music }}\left(\widehat{\theta}_{k}, \widehat{\phi}_{k}\right)=\left[\min _{\theta, \phi} \lambda_{\min }(\mathbf{B}(\theta, \phi))\right]^{-1} .
$$

From (18), the signals DOA estimation, that is, $\left(\theta_{k}, \phi_{k}\right)$, is got; the arrival angle function matrix $\Omega$ is herein achieved. It also can be seen that the signals DOA estimation is completely independent of polarization estimation and this method can decouple the DOA estimation from the polarization estimation. The estimation of $\widehat{\mathbf{B}}\left(\widehat{\theta}_{k}, \widehat{\phi}_{k}\right)=\boldsymbol{\Omega}^{\mathrm{H}} \mathbf{U}_{n} \mathbf{U}_{n}^{\mathrm{H}} \boldsymbol{\Omega}$ is acquired by using the estimates of $\boldsymbol{\Omega}$ and the DR-MUSIC polarization spectrum function is obtained. Consider

$$
\mathbf{P}_{\text {music }}\left(\widehat{\gamma}_{k}, \widehat{\eta}_{k}\right)=\frac{1}{\min _{\gamma, \eta}\left(\mathbf{g}^{\mathrm{H}}(\gamma, \eta) \mathbf{B}\left(\widehat{\theta}_{k}, \widehat{\phi}_{k}\right) \mathbf{g}(\gamma, \eta)\right)} .
$$

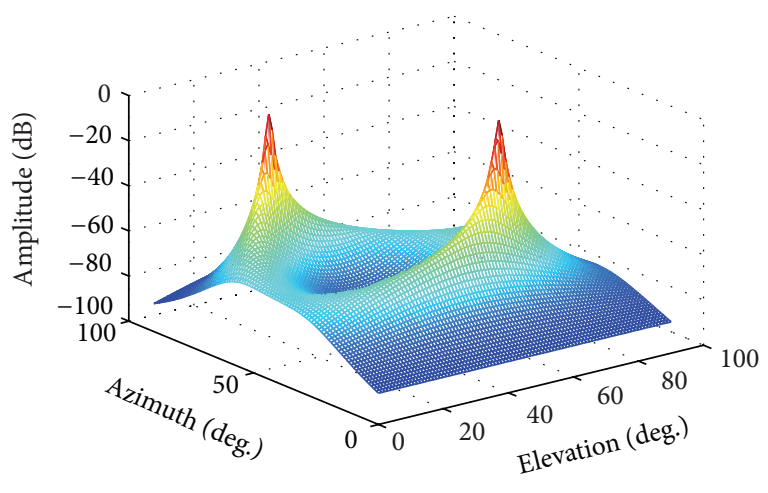

FIGURE 2: Spatial spectrum of our algorithm.

The polarization estimation is obtained by exerting a $2 \mathrm{D}$ search through the ranges of polarization parameters in (19). The proposed DR-MUSIC algorithm can reduce the dimensionality of search from a four-dimensional search to two two-dimensional searches; the amount of calculation is hence greatly deceased.

\section{Computational Complexity}

It seems that one straightforward way to estimate the DOA and polarization parameters of incident signals is to calculate the value of space spectrum corresponding to each direction and polarization state and then extract the location of the peaks. But this process invokes a search throughout the fourdimensional (4D) space (spanned by two DOA parameters and two polarization parameters). The computational complexity of $4 \mathrm{D}$ peak search is proportional with $\mathrm{L}^{4}$, where $\mathrm{L}$ is the number of angles to be searched. For the proposed method, it is roughly $2 \mathrm{~L}^{2}$ plus some additional calculations for the eigenvalues evaluations. This shows superiority of the proposed method in terms of computational time. Because of the vast amounts of computation, the above-mentioned $4 \mathrm{D}$ search is exhaustive and infeasible. Hence we did not do the $4 \mathrm{D}$ search experiment to compare with our DR-MUSIC experiment; this procedure is of no significance.

\section{Simulation Results}

Some simulations are conducted to evaluate the performances on DOA and polarization estimation by the proposed method. Two uncorrelated equal-powered signals with the following parameter values, $\left(\theta_{1}, \phi_{1}, \gamma_{1}, \eta_{1}\right)=$ $\left(30^{\circ}, 60^{\circ}, 20^{\circ}, 60^{\circ}\right)$ and $\left(\theta_{2}, \phi_{2}, \gamma_{2}, \eta_{2}\right)=\left(70^{\circ}, 20^{\circ}, 70^{\circ}, 40^{\circ}\right)$, impinge upon the uni-vector-sensor. 1024 snapshots are used for all simulation experiments. SNR is taken to be $15 \mathrm{~dB}$ from Figure 2 to Figure 5. In the first experiment, the DOA and polarization plan corresponding to the signal sources are represented for DR-MUSIC estimator in Figures 2 and 3, respectively.

The spatial spectrum picture of the DR-MUSIC algorithm is showed in Figure 2. It can be observed that the spatial 


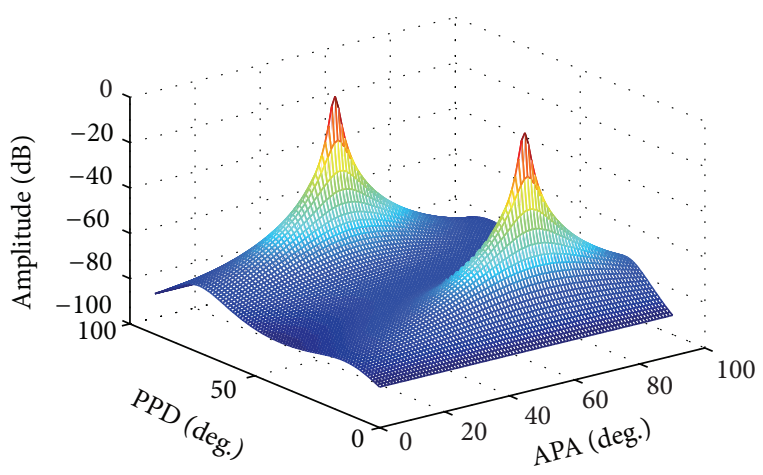

Figure 3: Polarization spectrum of our algorithm.

MUSIC search is accurate after decoupling the DOA estimation from the polarization estimation and the expected peaks were found. It can also be proved that the DR-MUSIC algorithm has superior suppression effect of sidelobe sources and high resolution. The polarization spectrum of the DRMUSIC algorithm is indicated in Figure 3. It is seen that DR-MUSIC can successfully detect the two signals, and the expected polarization spectrum peaks were found. The DR-MUSIC algorithm also has good suppression effect of sidelobe sources and high resolution. The corresponding course of polarization MUSIC search is herein correct.

In the second experiment, we consider a scenario with 100 independent trials running on the corresponding univector-sensor. The sets of values of the DOA and polarization variables have been represented in two-dimensional scatter diagrams (Figures 4 and 5). From Figures 4 and 5, it is shown that almost all estimated values are located in the vicinity of actual values. For example, consider signal one and the estimated value of elevation and azimuth range from the numerical ranges $\left(29.6^{\circ}, 30.4^{\circ}\right)$ and $\left(59.2^{\circ}, 60.8^{\circ}\right)$, respectively. Similarly, the estimated values of APA (auxiliary polarization angle) and PPD (polarization phase difference) range from the numerical range $\left(19.4^{\circ}, 20.6^{\circ}\right)$ and $\left(59.4^{\circ}, 60.6^{\circ}\right)$, respectively.

In the following experiments, we demonstrate the DOA and polarization estimation performance using the DRMUSIC algorithm with 100 independent trials running on the corresponding uni-vector-sensor. The simulation settings are kept unchanged except that SNR is set to range between 0 and $27 \mathrm{~dB}$.

In the third experiment, the solid line with circular and star data points in Figure 6, respectively, plots the estimation standard deviation of DOA and polarization, respectively, at various signal-to-noise ratio (SNR) levels, using the proposed DR-MUSIC method. For SNRs above $0 \mathrm{~dB}$, estimation standard deviations of DOA and polarization both decrease nearly linearly with increasing SNR dB values. The standard deviations of $\mathrm{DOA}$ and polarization are less than $1.5^{\circ}$ at $\mathrm{SNR}=15 \mathrm{~dB}$. Figure 7 plots the estimation RMSE of DOA and polarization, respectively, at different SNR levels, using the proposed DR-MUSIC method. For SNRs above $0 \mathrm{~dB}$, estimation RMSE of DOA and polarization both decrease

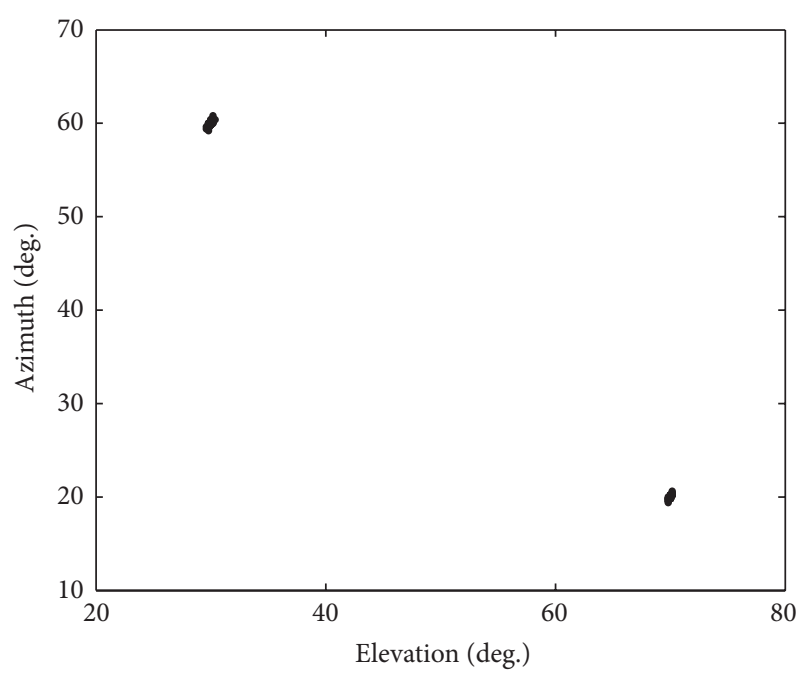

FIGURE 4: Scatter diagram of DOA.

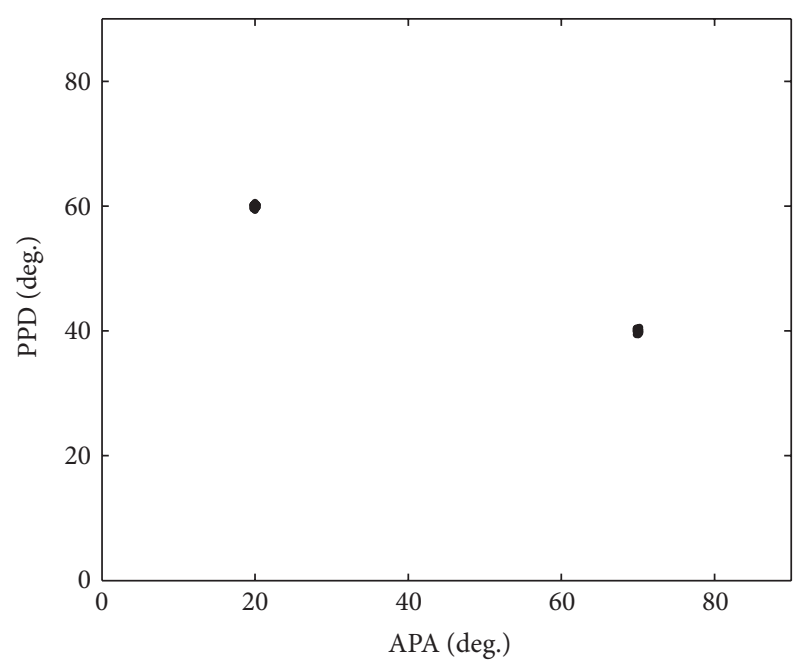

FIGURE 5: Scatter diagram of polarization.

nearly linearly with increasing SNR dB values. The RMSE of $\mathrm{DOA}$ and polarization are less than $1.5^{\circ}$ at $\mathrm{SNR}=15 \mathrm{~dB}$.

In the fourth experiment, we choose signal one as an example to illustrate the performance of the proposed DR-MUSIC algorithm. Comparison with the ESPRIT-based algorithm [13] is also made.

The dashed line with circular data points and the solid line with diamond data points in Figures 8, 9, 10, and 11 indicate the standard deviation of elevation, azimuth, APA (auxiliary polarization angle), and PPD (polarization phase difference), respectively. The results are estimated by ESPRIT and the proposed MUSIC method, at various signal-to-noise ratio (SNR) levels.

The performance of the proposed DR-MUSIC method is nearly the same as the uni-vector-sensor ESPRIT method [13]. Moreover, the standard deviations of azimuth, elevation, PPD, and APA are reduced evidently as the SNR increases using the DR-MUSIC method. However, the ESPRIT-based 


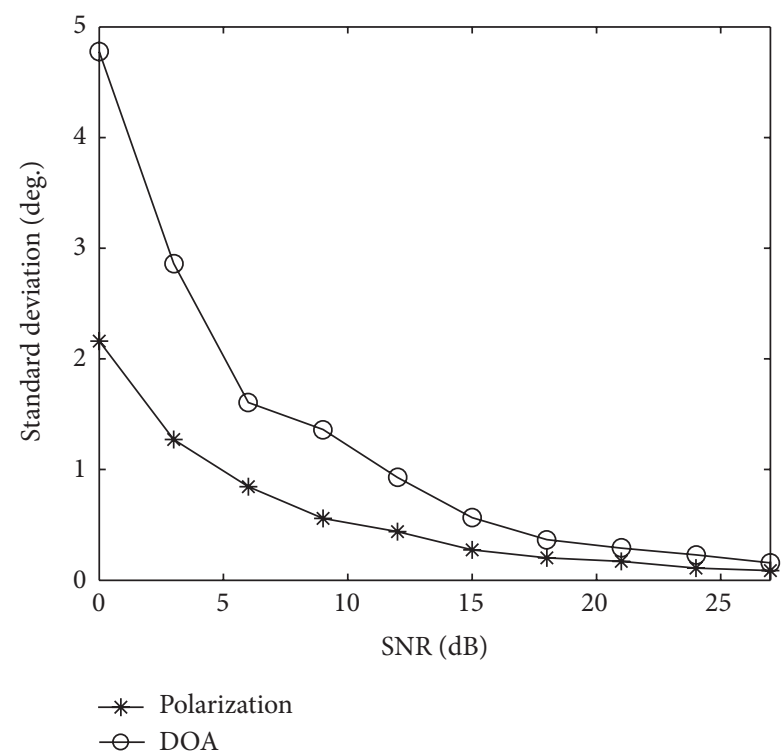

FIGURE 6: Standard deviation of DOA and polarization.

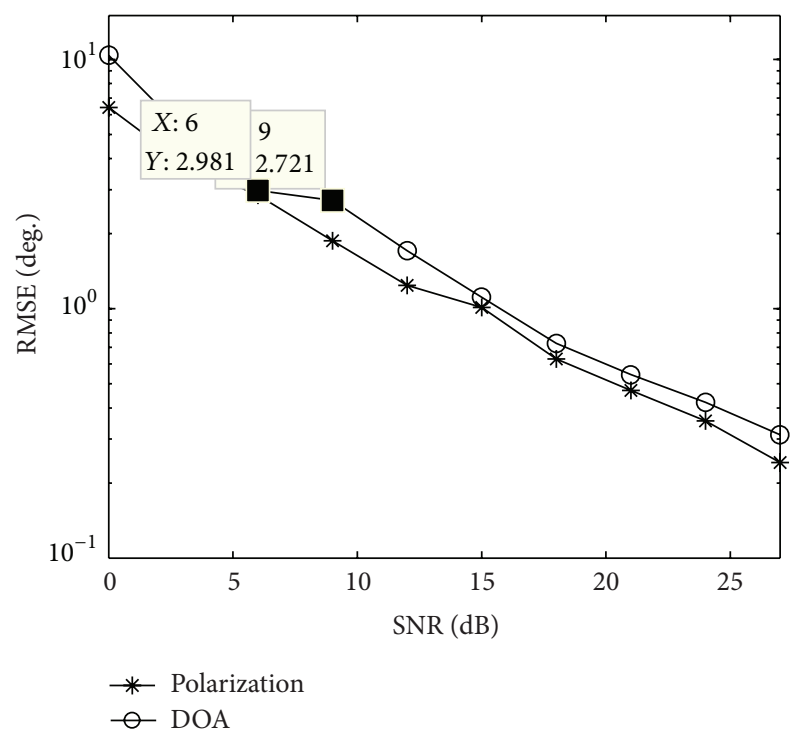

FIGURE 7: RMSE of DOA and polarization.

algorithm requires that the array geometric structure has the rotational invariant property, which severely restricts its application in engineering. In contrast, our proposed DRMUSIC algorithm not only does not need any specific array structure, but also can be directly applied to the $4 \mathrm{D}$ parameter estimation while the amount of calculation is twice as much as the 2D MUSIC algorithm. In the fifth experiment, the comparison of CRB and our proposed algorithm is given. In Figures 12, 13, 14, and 15, the virtual line and solid line with star data points, respectively, plot the estimation RMSE of azimuth, elevation, APA, and PPD, respectively, at various signal-to-noise ratio (SNR) levels, using the CRB method and the proposed DR-MUSIC method.

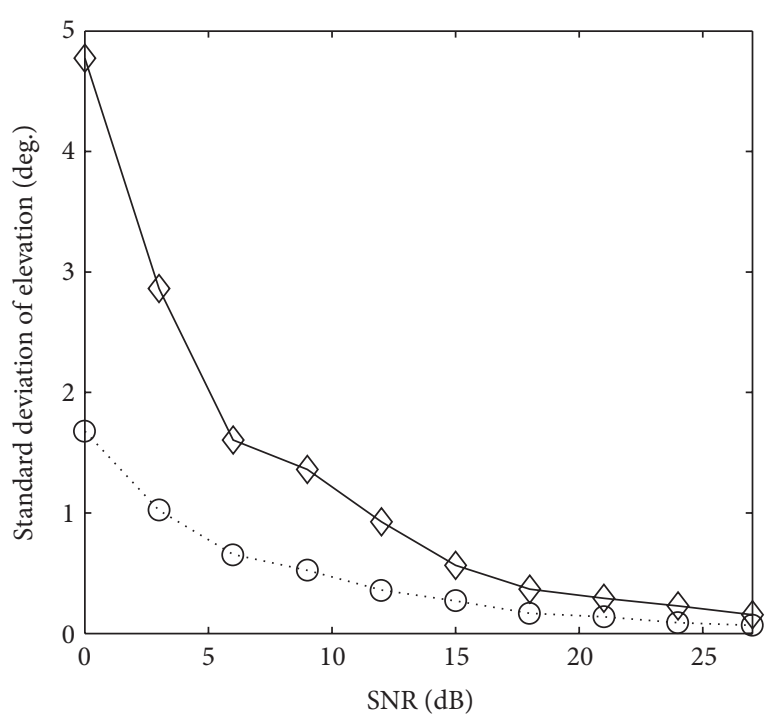

○. ESPRIT method

$\diamond$ MUSIC method

FIGURE 8: Standard deviation of elevation.

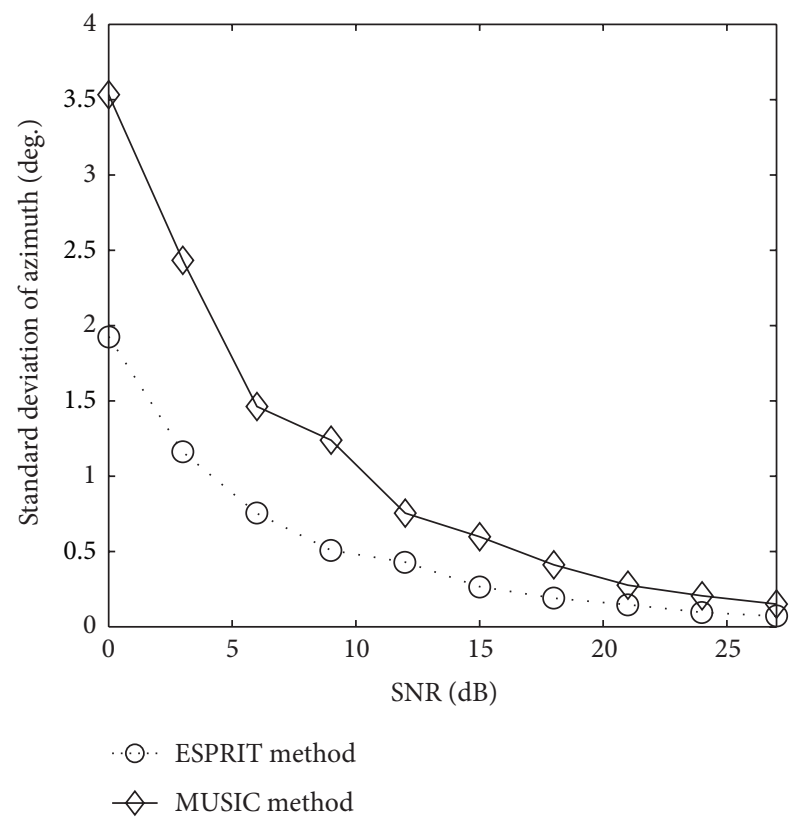

FIGURE 9: Standard deviation of azimuth.

It is clearly shown that our algorithm can work well. The performance of the proposed method is close to that of the CRB. For SNRs above $0 \mathrm{~dB}$, estimation RMSE of DOA and polarization both decrease nearly linearly with increasing SNR $\mathrm{dB}$ values. In the last experiment, we consider the probability of success of DOA and polarization parameter estimations. Here we keep the settings unchanged and set the relationship $|q-\hat{q}|<1.5^{\circ}$ to be the successful experiment, where $q$ denotes the true value and $\hat{q}$ represents the corresponding estimated value. The result is shown in Figure 16. 


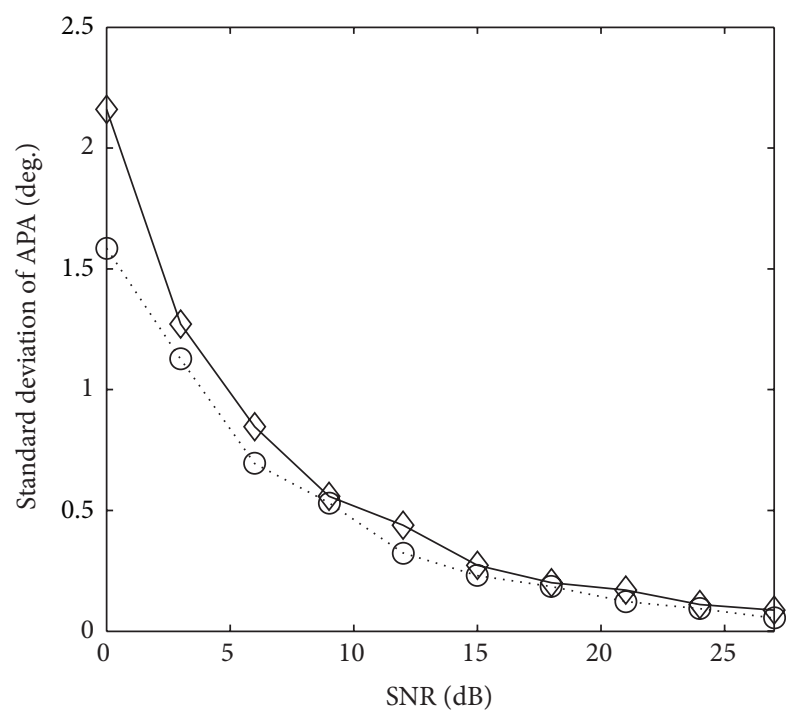

○. ESPRIT method

$\diamond$ MUSIC method

Figure 10: Standard deviation of APA.

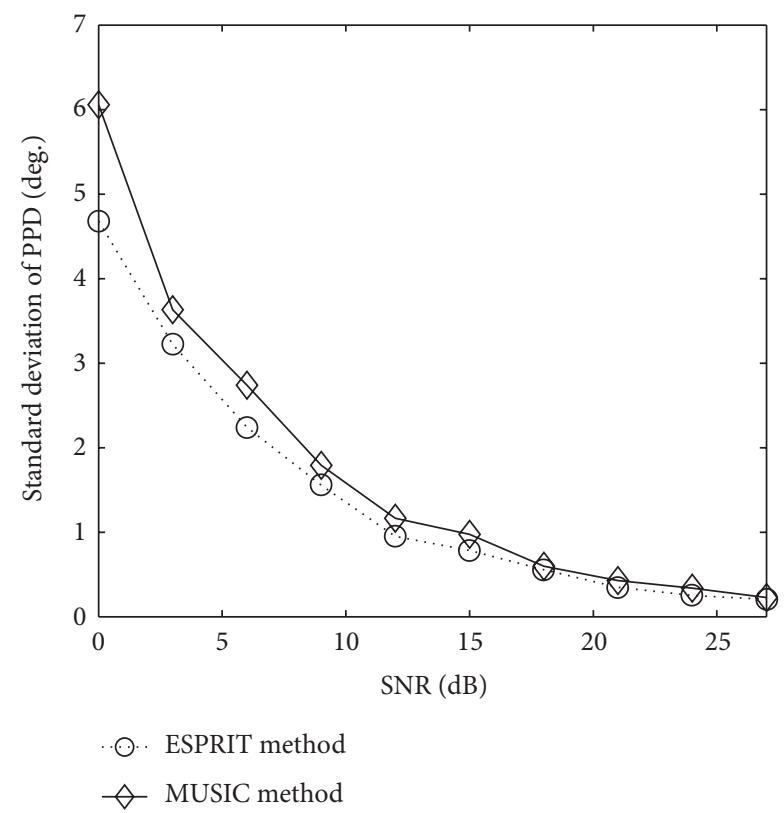

FIgURE 11: Standard deviation of PPD.

The solid line with circular and star data points in Figure 16, respectively, plots the probability of exact recovery of DOA and polarization, respectively, at different SNR levels, using the proposed DR-MUSIC method. We can see from Figure 16 that the probability of exact recovery is nearly at $100 \%$ at SNR level of $15 \mathrm{~dB}$.

From the above analysis, it can be seen that DR-MUSIC provides the superior estimation accuracy. Compared with the self-initiating MUSIC algorithm in [28], the proposed DR-MUSIC algorithm requires only two two-dimensional search operations. The proposed algorithm also has

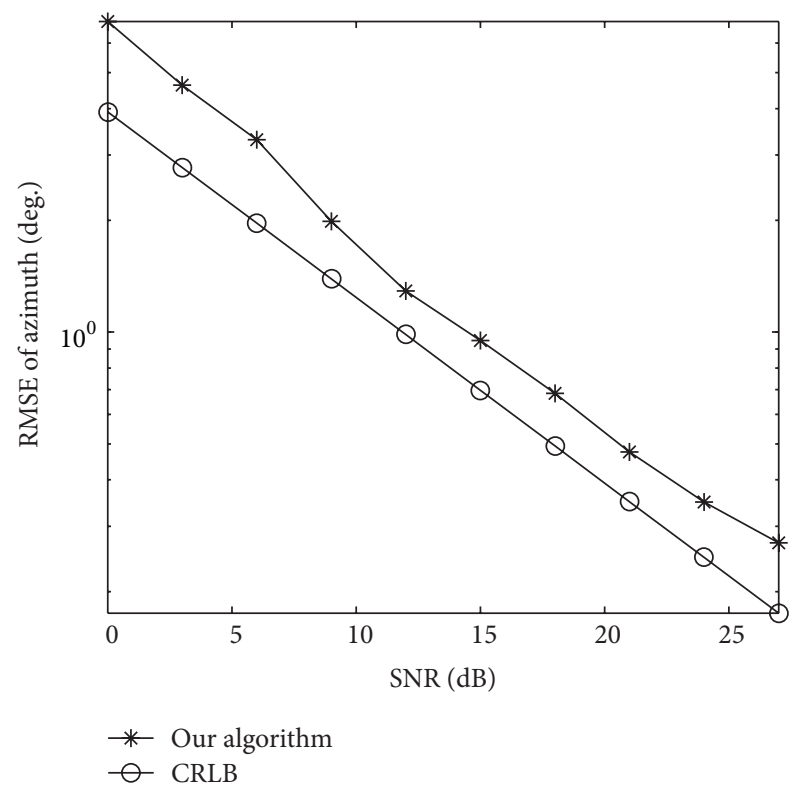

FIGURE 12: RMSE of azimuth versus SNR.

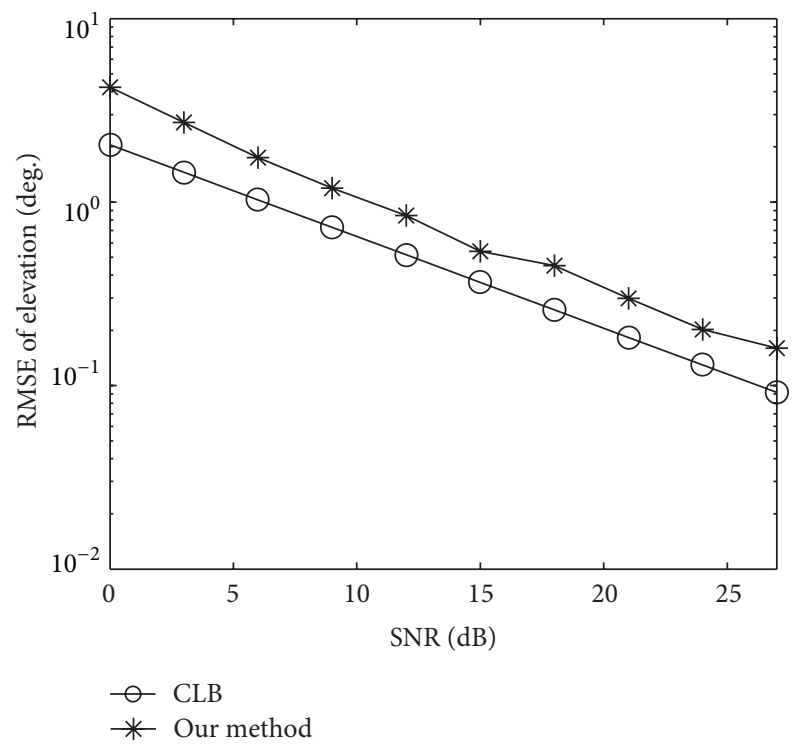

FIgURE 13: RMSE of elevation versus SNR.

the advantage of DOA and polarization automatic matching. However, the self-initiating MUSIC algorithm must use ESPRIT on pairs of vector sensors to self-generate coarse estimates of the arrival angles to start off its MUSIC-based iterative search.

\section{Conclusion}

In this paper, a novel dimensionality reduction MUSICbased algorithm for estimating the DOA and polarization has been proposed, which is directly applied to the univector-sensor case. The signal steering vector is decomposed into the product of the arrival angle function matrix and 


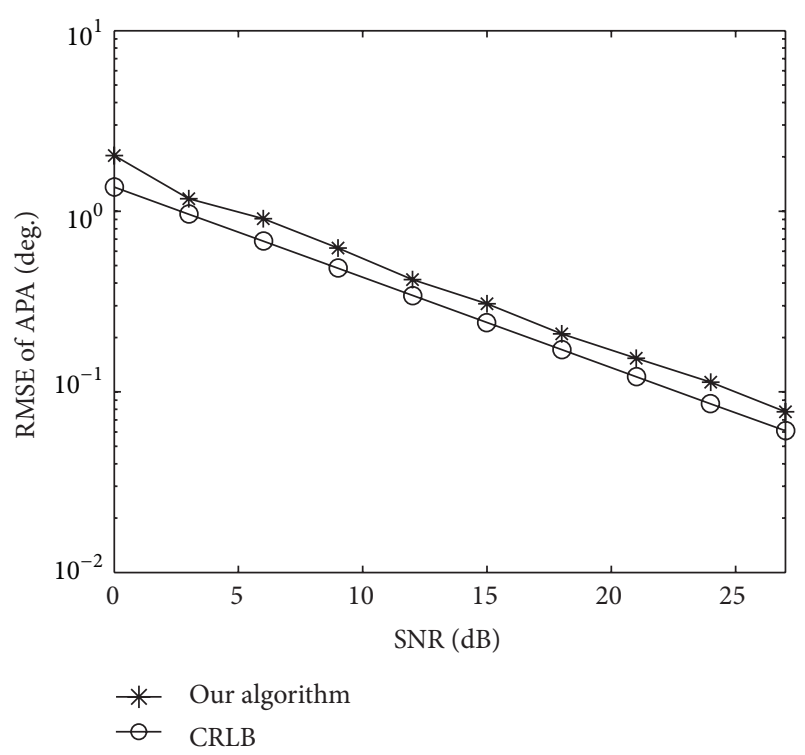

FIGURE 14: RMSE of APA versus SNR.

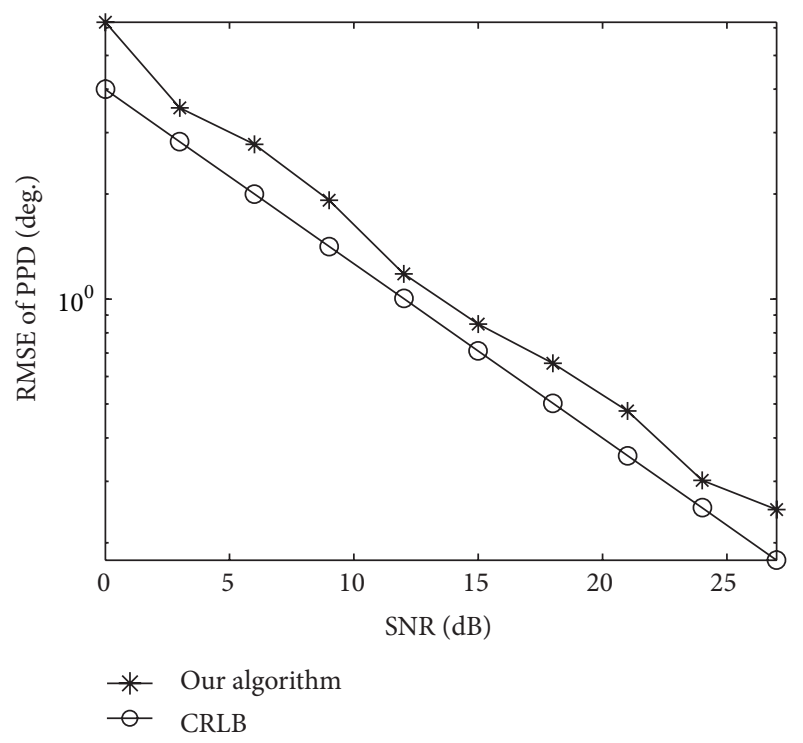

FIGURE 15: RMSE of PPD versus SNR.

polarization function vector. Using the feature that the 2norm of polarization function vector is constant, the joint spectrum in spatial and polarization domains is converted to the Rayleigh-Ritz ratio form. By deploying the Rayleigh-Ritz theorem, the joint spectrum can decouple the spatial (DOA, i.e., azimuth and elevation) spectrum and the polarization (auxiliary polarization angle and polarization phase difference) spectrum, resulting in two degrees of dimensionality reduction in MUSICs search procedure. This method is useful for the MUSIC-based dimensionality reduction for six-component electromagnetic vector sensor array where the customary MUSIC search is extremely computation exhaustive. This permits the extension of uni-vector-sensor

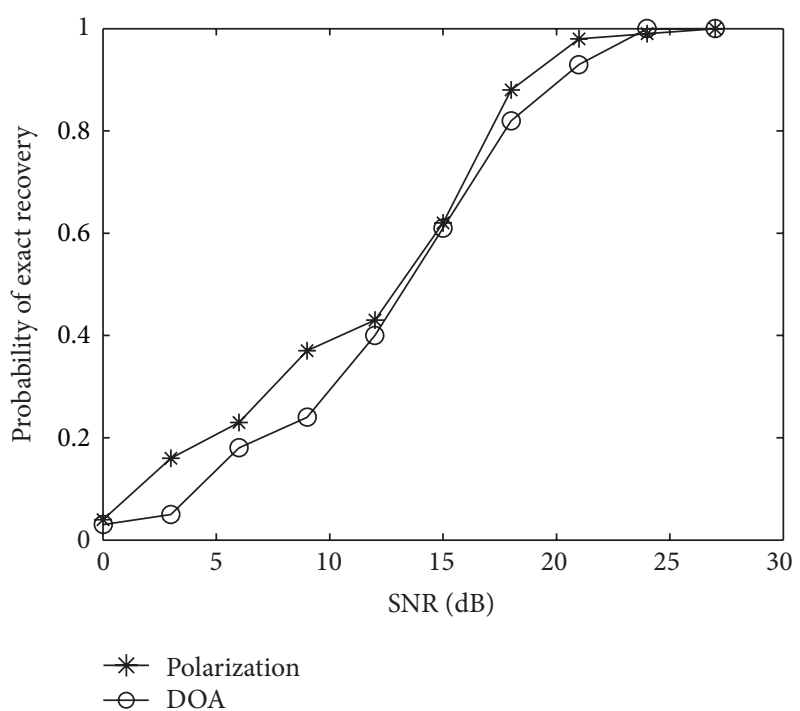

FIGURE 16: Probability of exact recovery of DOA and polarization.

MUSIC algorithm to an arbitrary number of components of a vector sensor array.

\section{Conflict of Interests}

The authors declare that there is no conflict of interests regarding the publication of this paper.

\section{Acknowledgments}

This work was supported by the National Natural Science Foundation of China $(61201295,61231017)$ and the Fundamental Research Funds for the Central Universities (K5051307017). The authors would like to thank the anonymous reviewers and the associated editor for their valuable comments and suggestions that improved the clarity of this paper.

\section{References}

[1] T. Li and A. Nehorai, "Maximum likelihood direction-ofarrival estimation of underwater acoustic signals containing sinusoidal and random components," IEEE Transactions on Signal Processing, vol. 59, no. 11, pp. 5302-5314, 2011.

[2] R. Keizer, T. Bronez, and J. Creekmore, "An approximate maximum likelihood algorithm for direction finding with diversely polarized arrays," in Proceedings of the IEEE Conference Record on Communications on the Move-Military Communications Conference (MILCOM '93), vol. 1, pp. 349-353, Boston, Mass, USA, October 1993.

[3] X. F. Zhang and D. Z. Xu, "Blind PARAFAC signal detection for polarization sensitive array," EURASIP Journal on Advances in Signal Processing, vol. 2007, Article ID 12025, 7 pages, 2007.

[4] B. Hochwald and A. Nehorai, "Identifiability in array processing models with vector-sensor applications," IEEE Transactions on Signal Processing, vol. 44, no. 1, pp. 83-95, 1996. 
[5] X. Zhang, Y. Shi, and D. Xu, "Novel blind joint direction of arrival and polarization estimation for polarization-sensitive uniform circular array," Progress in Electromagnetics Research, vol. 86, pp. 19-37, 2008.

[6] X. F. Zhang and D. Z. Xu, "Deterministic blind beamforming for electromagnetic vector sensor array," Progress in Electromagnetics Research, vol. 84, pp. 363-377, 2008.

[7] Q. Cheng and Y. Hua, "Performance analysis of the MUSIC and pencil-MUSIC algorithms for diversely polarized array," IEEE Transactions on Signal Processing, vol. 42, no. 11, pp. 3150-3165, 1994.

[8] R. O. Schmidt, "Multiple emitter location and signal parameter estimation," IEEE Transactions on Antennas and Propagation, vol. AP-34, no. 3, pp. 276-280, 1986.

[9] Y. Hua, "Pencil-MUSIC algorithm for finding two-dimensional angles and polarizations using crossed dipoles," IEEE Transactions on Antennas and Propagation, vol. 41, no. 3, pp. 370-376, 1993.

[10] M. D. Zoltowski and K. T. Wong, "Closed-form eigenstructurebased direction finding using arbitrary but identical subarrays on a sparse uniform Cartesian array grid," IEEE Transactions on Signal Processing, vol. 48, no. 8, pp. 2205-2210, 2000.

[11] M. D. Zoltowski and K. T. Wong, "ESPRIT-based 2-D direction finding with a sparse uniform array of electromagnetic vector sensors," IEEE Transactions on Signal Processing, vol. 48, no. 8, pp. 2195-2204, 2000.

[12] K. T. Wong and M. D. Zoltowski, "Closed-form direction finding and polarization estimation with arbitrarily spaced electromagnetic vector-sensors at unknown locations," IEEE Transactions on Antennas and Propagation, vol. 48, no. 5, pp. 671-681, 2000.

[13] K. T. Wong and M. D. Zoltowski, "Uni-vector-sensor ESPRIT for multisource azimuthelevation, and polarization estimation," IEEE Transactions on Antennas and Propagation, vol. 45, no. 10, pp. 1467-1474, 1997.

[14] K. T. Wong and M. D. Zoltowski, "Polarization diversity and extended aperture spatial diversity to mitigate fading-channel effects with a sparse array of electric dipoles or magnetic loops," in Proceedings of the IEEE International Electric Vehicle Conference, pp. 1163-1167, 1997.

[15] K. T. Wong and M. D. Zoltowski, "High accuracy 2D angle estimation with extended aperture vector sensor arrays," in Proceedings of the IEEE International Conference on Acoustics, Speech, and Signal Processing (ICASSP '96), vol. 5, pp. 27892792, Atlanta, Ga, USA, May 1996.

[16] J. Li and R. T. Compton Jr., "Angle and polarization estimation using ESPRIT with a polarization sensitive array," IEEE Transactions on Antennas and Propagation, vol. 39, no. 9, pp. 1376-1383, 1991.

[17] J. Li and R. T. Compton Jr., "Two-dimensional angle and polarization estimation using the ESPRIT algorithm," IEEE Transactions on Antennas and Propagation, vol. 40, no. 5, pp. 550-555, 1992.

[18] J. Li and R. T. Compton Jr., "Angle estimation using a polarization sensitive array," IEEE Transactions on Antennas and Propagation, vol. 39, no. 10, pp. 1539-1543, 1991.

[19] K. T. Wong and M. D. Zoltowski, "Direction-finding with sparse rectangular dual-size spatial invariance array," IEEE Transactions on Aerospace and Electronic Systems, vol. 34, no. 4, pp. 1320-1336, 1998.
[20] K. T. Wong, "Direction finding/polarization estimationDipole and/or loop triad(s)," IEEE Transactions on Aerospace and Electronic Systems, vol. 37, no. 2, pp. 679-684, 2001.

[21] K. T. Wong and M. D. Zoltowski, "Closed-form directionfinding with arbitrarily spaced electromagnetic vector-sensors at unknown locations," in Proceedings of the IEEE International Conference on Acoustics, Speech and Signal Processing (ICASSP '98), vol. 4, pp. 1949-1952, May 1998.

[22] K. T. Wong and M. D. Zoltowski, "Root-MUSIC-based azimuth-elevation angle-of-arrival estimation with uniformly spaced but arbitrarily oriented velocity hydrophones," IEEE Transactions on Signal Processing, vol. 47, no. 12, pp. 3250-3260, 1999.

[23] K. T. Wong and A. K.-Y. Lai, "Inexpensive upgrade of basestation dumb antennas by two magnetic loops for "blind" adaptive downlink beamforming," IEEE Antennas and Propagation Magazine, vol. 47, no. 1, pp. 189-193, 2005.

[24] X. Gong, Z. Liu, Y. Xu, and M. I. Ahmad, "Direction-of-arrival estimation via twofold mode-projection," Signal Processing, vol. 89, no. 5, pp. 831-842, 2009.

[25] X. Yuan, K. T. Wong, and K. Agrawal, "Polarization estimation with a dipole-dipole pair, a dipole-loop pair, or a loop-loop pair of various orientations," IEEE Transactions on Antennas and Propagation, vol. 60, no. 5, pp. 2442-2452, 2012.

[26] A. Nehorai and E. Paldi, "Vector-sensor array processing for electromagnetic source localization," IEEE Transactions on Signal Processing, vol. 42, no. 2, pp. 376-398, 1994.

[27] J. Li, "Direction and polarization estimation using arrays with small loops and short dipoles," IEEE Transactions on Antennas and Propagation, vol. 41, no. 3, pp. 379-386, 1993.

[28] K. T. Wong and M. D. Zoltowski, "Self-Initiating MUSICBased direction finding and polarization estimation in spatiopolarizational beamspace," IEEE Transactions on Antennas and Propagation, vol. 48, no. 8, pp. 1235-1245, 2000.

[29] K. T. Wong, L. Li, and M. D. Zoltowski, "Root-MUSIC-based direction-finding and polarization estimation using diversely polarized possibly collocated antennas," IEEE Antennas and Wireless Propagation Letters, vol. 3, no. 1, pp. 129-132, 2004.

[30] R. Guo, X. P. Mao, S. B. Li, Z. M. Lin, W. B. Deng, and P. Jiang, "Analysis for 4-D fast polarization MUSIC algorithm," in Proceedings of the IEEE Radar Conference (RADAR '13), pp. 1-4, Ottawa, Canada, April-May 2013.

[31] R. Guo, X. P. Mao, S. B. Li, and Z. M. Lin, "Fast fourdimensional joint spectral estimation with array composed of diversely polarized elements," in Proceedings of the IEEE Radar Conference, pp. 919-923, 2012.

[32] X. Zhang, C. Chen, J. Li, and D. Xu, "Blind DOA and polarization estimation for polarization-sensitive array using dimension reduction MUSIC," Multidimensional Systems and Signal Processing, vol. 25, no. 1, pp. 67-82, 2014.

[33] R. Goossens and H. Rogier, "Direction-of-arrival and polarization estimation with uniform circular arrays in the presence of mutual coupling," AEU-International Journal of Electronics and Communications, vol. 62, no. 3, pp. 199-206, 2008.

[34] A. H. Roger and R. J. Charles, Matrix Analysis, Cambridge University Press, New York, NY, USA, 2012. 


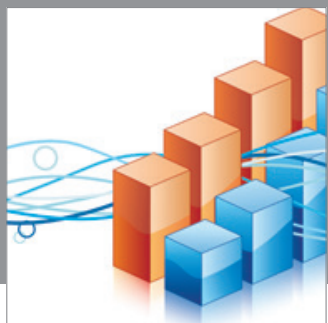

Advances in

Operations Research

mansans

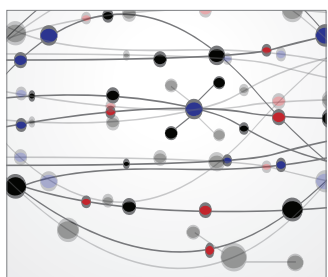

The Scientific World Journal
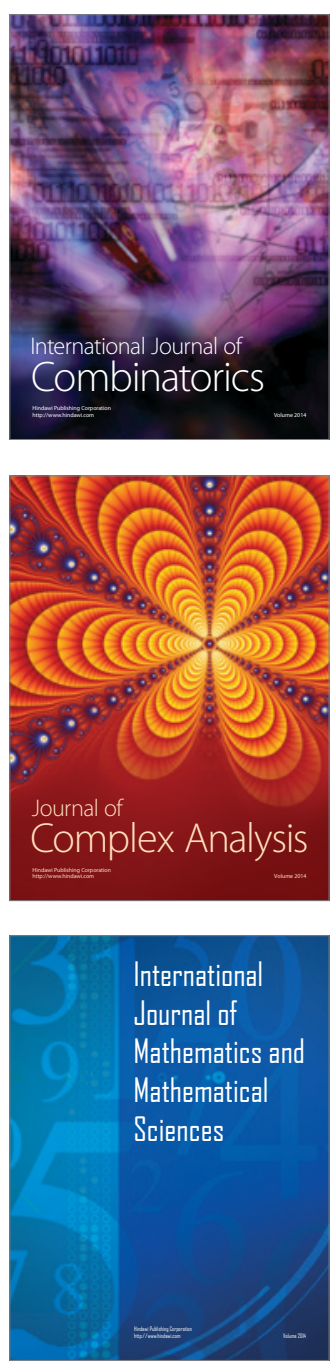
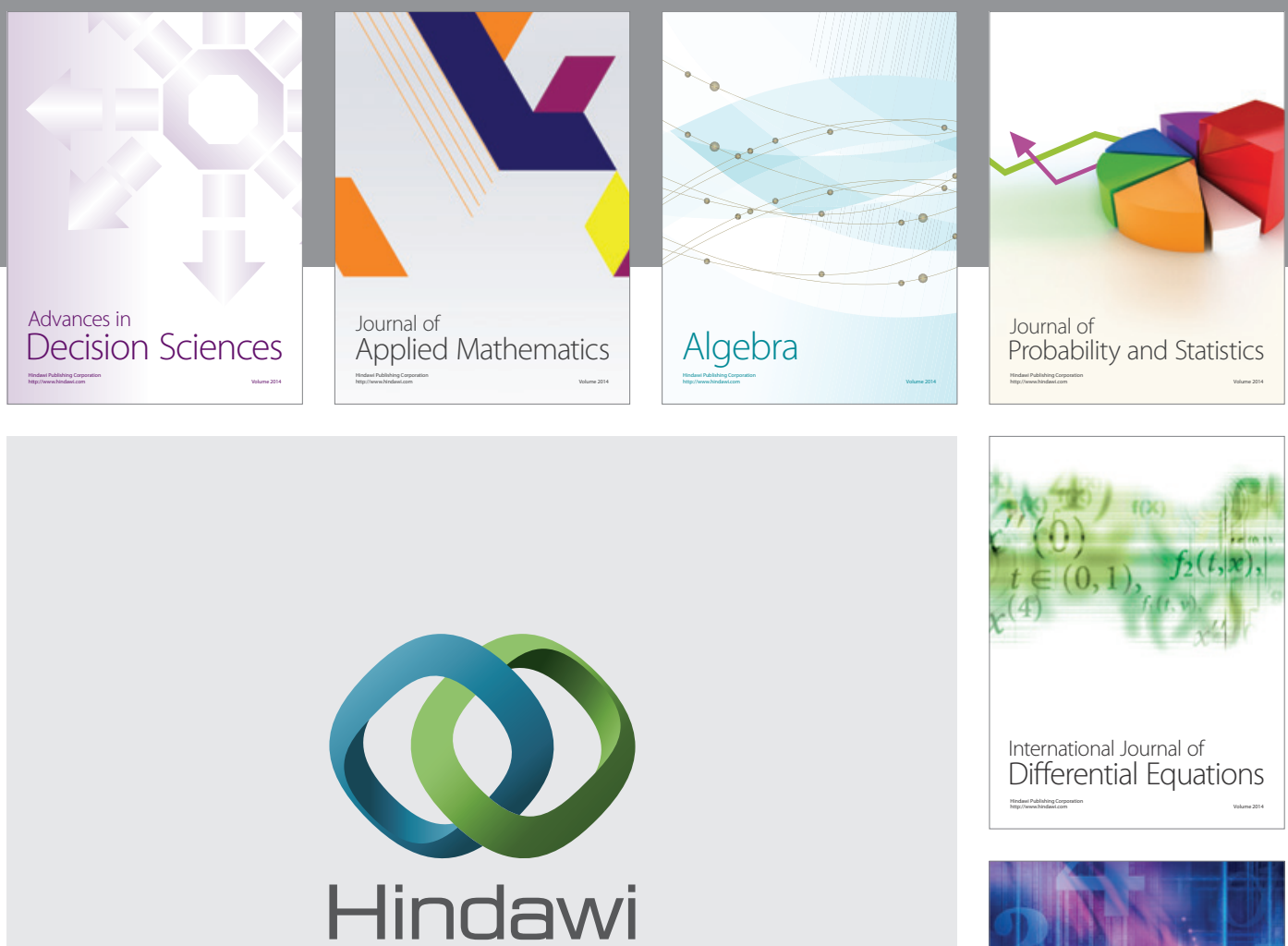

Submit your manuscripts at http://www.hindawi.com
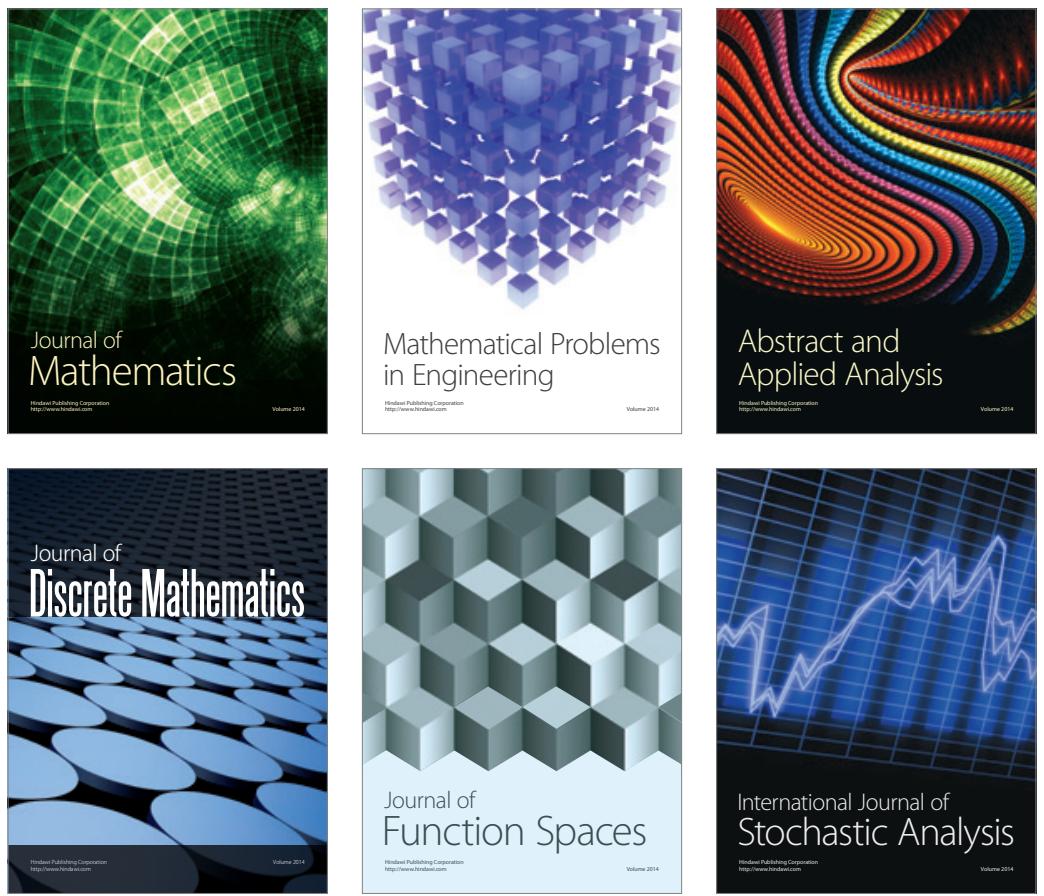

Journal of

Function Spaces

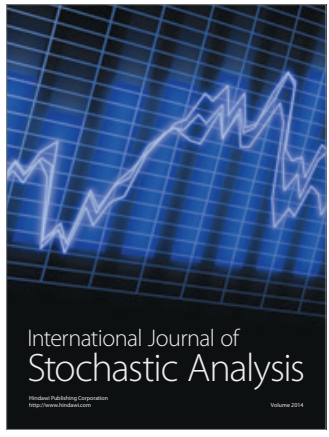

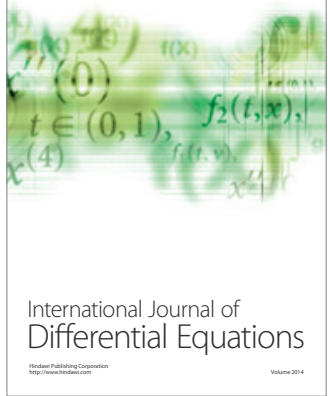
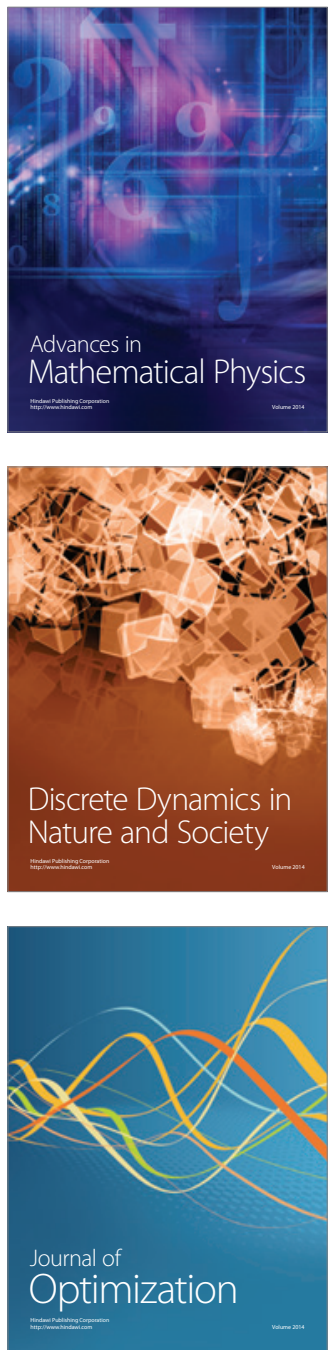